\title{
Genotoxic effects of occupational exposure to lead and influence of polymorphisms in genes involved in lead toxicokinetics and in DNA repair
}

\author{
Julia García-Lestón a,b,1 , Joana Roma-Torres ${ }^{\text {c,d, } 1}{ }^{\text {, Maria Vilares }}{ }^{\mathrm{e}}$, Rui Pinto ${ }^{\mathrm{f}}$, João Prista ${ }^{\mathrm{d}}$, \\ João Paulo Teixeira ${ }^{\mathrm{c}}$, Olga Mayan ${ }^{\mathrm{c}}$, João Conde $^{\mathrm{g}}$, Marta Pingarilho ${ }^{\mathrm{g}}$, Jorge Francisco Gaspar ${ }^{\mathrm{g}}$, \\ Eduardo Pásaro ${ }^{\mathrm{a}}$, Josefina Méndez ${ }^{\mathrm{b}}$, Blanca Laffon ${ }^{\mathrm{a}, *}$ \\ a Toxicology Unit, Department of Psychobiology, University of A Coruña, Edificio de Servicios Centrales de Investigación, Campus Elviña, 15071-A Coruña, Spain \\ ${ }^{\mathrm{b}}$ Department of Cell and Molecular Biology, University of A Coruña, Facultad de Ciencias, Campus da Zapateira, 15071-A Coruña, Spain \\ ${ }^{c}$ National Institute of Health, Environmental Health Department, Rua Alexandre Herculano 321, 4000-055-Porto, Portugal \\ d Escola Nacional de Saúde Pública, Universidade Nova de Lisboa, Av. Padre Cruz, 1600-560 Portugal \\ e National Institute of Health, Department of Health and health-promoting chronic diseases, Unit of Research and Development, Av. Padre Cruz, 1649-061 Lisboa, Portugal \\ f iMed.UL, Research Institute for Medicines and Pharmaceutical Sciences, Faculty of Pharmacy, University of Lisbon, Av. Prof. Gama Pinto, 1649-003 Lisboa, Portugal \\ ${ }^{g}$ Department of Genetics, Faculdade de Ciências Médicas, Universidade Nova de Lisboa, Rua da Junqueira 100, P-1349-008 Lisbon, Portugal
}

\section{A R T I C L E I N F O}

\section{Article history:}

Received 22 November 2011

Accepted 3 March 2012

Available online 30 March 2012

\section{Keywords:}

Lead

Genotoxicity

T-cell receptor mutation assay

Micronucleus test

Comet assay

Genetic polymorphisms

\begin{abstract}
A B S T R A C T
Lead is still widely used in many industrial processes and is very persistent in the environment. Although toxic effects caused by occupational exposure to lead have been extensively studied, there are still conflicting results regarding its genotoxicity. In a previous pilot study we observed some genotoxic effects in a population of lead exposed workers. Thus, we extended our study analysing a larger population, increasing the number of genotoxicity endpoints, and including a set of 20 genetic polymorphisms related to lead toxicokinetics and DNA repair as susceptibility biomarkers. Our population comprised 148 workers from two Portuguese factories and 107 controls. The parameters analysed were: blood lead levels (BLL) and $\delta$-aminolevulinic acid dehydratase (ALAD) activity as exposure biomarkers, and T-cell receptor (TCR) mutation assay, micronucleus (MN) test, comet assay and OGG1-modified comet assay as genotoxicity biomarkers. Lead exposed workers showed markedly higher BLL and lower ALAD activity than the controls, and significant increases of TCR mutation frequency (TCR-Mf), MN rate and DNA damage. Oxidative damage did not experience any significant alteration in the exposed population. Besides, significant influence was observed for VDR rs1544410 polymorphism on BLL; APE1 rs1130409 and LIG4 rs1805388 polymorphisms on TCR-Mf; MUTYH rs3219489, XRCC4 rs28360135 and LIG4 rs1805388 polymorphisms on comet assay parameter; and OGG1 rs1052133 and XRCC4 rs28360135 polymorphisms on oxidative damage. Our results showed genotoxic effects related to occupational lead exposure to levels under the Portuguese regulation limit of $70 \mu \mathrm{g} / \mathrm{dl}$. Moreover, a significant influence of polymorphisms in genes involved in DNA repair on genotoxicity biomarkers was observed.
\end{abstract}

(C) 2012 Elsevier Ltd. All rights reserved.
Abbreviations: A, alanine; ALAD, $\delta$-aminolevulinic acid dehydratase; ALADact, activity of aminolevulinic acid dehydratase; BER, base excision repair; BLL, blood lead levels; C, cysteine; D, aspartatic acid; DNAT, DNA in the tail of the comet; E, glutamic acid; FITC, fluorescein-isothiocyanate; $\mathrm{H}$, histidine; HR, homologous recombination; IARC, International Agency for Research on Cancer; I, isoleucine; K, lysine; M, methionine; MN, micronucleus; N, asparagine; NHEJ, non-homologous end-joining; OSHA, Occupational Safety and Health Administration; PE, phycoerytrin; P, proline; Q glutamine; R, arginine; S, serine; T, threonine; TCR, T-cell receptor; TCR-Mf, T-cell receptor mutation frequency; V, valine; VDR, vitamin D receptor; $\mathrm{W}$, tryptophan.

* Corresponding author at: Toxicology Unit, University of A Coruña, Edificio de Servicios Centrales de Investigación, Campus Elviña s/n, 15071-A Coruña, Spain. Tel.: + 34981 167000; fax: + 34981167172.

E-mail address: blaffon@udc.es (B. Laffon).

1 These authors contributed equally to this work.

\section{Introduction}

Although the toxic effect of lead has been known for centuries, it is still widely used in many industrial processes that include mining, household renovation, battery and automobile manufacturing, and production of plastics, ceramics, paints and pigments that may involve human exposure to this metal (Kasuba et al., 2010). Due to its wide distribution and long persistence in the environment, exposure to lead is a great hazard for human health. In fact, the International Agency for Research on Cancer (IARC) classified it as possible human carcinogen (group 2B) (IARC, 1987) and its inorganic compounds as probable human carcinogens (group 2A) (IARC, 2006).

Adverse health effects caused by lead have long been recognized. It is known that exposure to this heavy metal adversely affects human health by producing toxicity in several organs and systems including the cardiovascular, renal, immune and reproductive systems, as well 
as on bones and teeth (García-Lestón et al., in press; Mansouri and Cauli, 2009). Although toxic effects caused by occupational exposure to lead have been extensively studied, there are still conflicting results regarding its genotoxicity (reviewed in García-Lestón et al., 2010). Several mechanisms have been suggested to be involved, such as induction of cellular immunity and oxidative stress, inhibition of DNA metabolism and repair, and formation of DNA and/or protein crosslinks (Mendez-Gomez et al., 2008).

In a previous study, we evaluated the genotoxic effects of occupational exposure to lead in a group of 70 Portuguese workers compared with 38 controls. The results revealed a significant increase in the mutation frequency of T-cell receptor (TCR) in the exposed individuals, and raised the possibility that some lifestyle factors could influence the extent of damage (García-Lestón et al., 2011). Nevertheless, no modifications in DNA damage (evaluated by means of the comet assay) were associated with the exposure, and no clear effect was observed for vitamin D receptor (VDR) genetic polymorphism on the parameters evaluated. Given these results, and the relevance of genotoxic events for the outcome of cancer, since they are tightly related to the first stages of carcinogenesis, we decided to extend our study. With this aim, we analysed a larger population, increased the number of genotoxicity endpoints, and included a set of genetic polymorphisms related to lead toxicokinetics and DNA repair as susceptibility biomarkers.

Thus, we evaluated the genotoxic effects of occupational lead exposure in a population of 148 workers from two Portuguese factories and 107 controls. The exposure was evaluated by analysing blood lead levels (BLL) and $\delta$-aminolevulinic acid dehydratase (ALAD) activity. TCR mutation assay, micronucleus (MN) test, comet assay and OGG1-modified comet assay, were performed to assess the genotoxicity. Besides, two polymorphisms in genes involved in lead toxicokinetics (VDR and $A L A D)$, and 18 polymorphisms in genes involved in base excision repair (BER) pathway (XRCC1, APE1, MUTYH, PARP1, MPG, PARP4, OGG1), nonhomologous end-joining (NHEJ) repair pathway (XRCC4, LIG4, XRCC5) and homologous recombination (HR) repair pathway (XRCC2, XRCC3, RAD51, NBS1) were genotyped as susceptibility biomarkers.

\section{Materials and methods}

\subsection{Subjects and biological samples}

Peripheral blood samples were obtained from 255 males and classified into two groups according to lead exposure. The first group consisted of 148 workers from two plants in Portugal that use inorganic lead in the workplace; plant 1 produce chemical substances and plant 2 manufacture lead-acid batteries. The second one comprised 107 controls involved in administrative and commercial functions in companies not using lead. Ethical approval from the National Institute of Health Ethical Board and individual informed consents were obtained. All the individuals participating in the study were asked to complete a questionnaire whereby relevant information on age, lifestyle factors (namely smoking and alcohol consumption habits), health conditions, and occupational history was obtained. The characteristics of the study population are shown in Table 1 . In order to ensure a blind study, all samples were coded just after collection.

\subsection{Blood lead levels}

Blood lead levels (BLL) were determined in samples collected in EDTA containers by electrothermal atomic absorption spectrometry as described in García-Lestón et al. (2011).

\subsection{ALAD activity}

A standardized European method (Far Diagnostics, kit n³602 (Berlin and Schaller, 1974)) was used to determine erythrocyte
Table 1

Characteristics of the study population. $P$ value refers to comparison between controls and exposed, or between factory 1 and factory 2 .

\begin{tabular}{|c|c|c|c|}
\hline & Controls & Exposed & $P$ value \\
\hline Total & 107 & 148 & \\
\hline Factory 1 & & 89 & \\
\hline Factory 2 & & 59 & \\
\hline Time of exposure (years) ${ }^{a}$ & & $19.54 \pm 0.94$ & \\
\hline Factory 1 & & $15.9 \pm 10.9$ & $<0.001^{\mathrm{c}}$ \\
\hline Factory 2 & & $25.0 \pm 10.1$ & \\
\hline Age (years) ${ }^{\mathrm{a}}$ & $43.1 \pm 11.8$ & $47.8 \pm 9.4$ & $<0.001^{\mathrm{c}}$ \\
\hline Factory 1 & & $46.3 \pm 10.3$ & $0.013^{c}$ \\
\hline Factory 2 & & $50.2 \pm 7.3$ & \\
\hline Smokers & $18(16.8 \%)$ & $60(40.5 \%)$ & $<0.001^{\text {b }}$ \\
\hline Factory 1 & & $42(47.2 \%)$ & $0.060^{\mathrm{b}}$ \\
\hline Factory 2 & & $18(30.5 \%)$ & \\
\hline No. cigarettes/day ${ }^{\mathrm{a}}$ & $14.9 \pm 11.1$ & $19.5 \pm 11.7$ & $0.146^{\mathrm{b}}$ \\
\hline Factory 1 & & $17.6 \pm 10.9$ & $0.078^{\mathrm{c}}$ \\
\hline Factory 2 & & $23.8 \pm 12.5$ & \\
\hline
\end{tabular}

ALAD activity. This parameter was expressed as units ALAD/ml erythrocytes $(1 \mathrm{U} / \mathrm{ml}$ corresponding to the substrate-5-aminolevulinatenanomoles transformed per minute and per erythrocytes $\mathrm{ml}$ ). Analyses were performed within $24 \mathrm{~h}$ of sampling because of ALAD instability.

\subsection{Leukocyte isolation}

BD Vacutainer ${ }^{\mathrm{TM}} \mathrm{CPT}^{\mathrm{TM}}$ Cell Preparation Tubes with sodium heparin (Becton Dickinson) were used for the isolation of mononuclear leukocytes following manufacturer's instructions. Cells were used in fresh for TCR mutation assay and MN test, or frozen for the comet assay (standard and OGG1-modified). In this last case, they were suspended in freezing medium (50\% foetal calf serum, 40\% RPMI 1640, $10 \%$ DMSO) to obtain $10^{7}$ cells $/ \mathrm{ml}$, and stored at $-80^{\circ} \mathrm{C}$ in a Nalgene ${ }^{\circledR}$ Cryo $1{ }^{\circ} \mathrm{C}$ Freezing Container (Nalgene Nunc International), until the time of analysis.

\subsection{TCR mutation assay}

TCR mutation assay was performed in duplicate by a flow cytometry methodology following Akiyama et al. (1995). Briefly, mononuclear leukocytes were stained with 7-amino-actinomycin $\mathrm{D}$ to gate out dead cells, and with fluorescein-isothiocyanate (FITC)-labeled anti-CD3 and phycoerytrin (PE)-labeled anti-CD4 antibodies (Becton Dickinson). A minimum of $2.5 \times 10^{5}$ lymphocyte gated events were acquired in a FACSCalibur flow cytometer with Cell Quest Pro software (Becton Dickinson). TCR mutation frequencies (TCR-Mf) were calculated as the number of events in the mutant cell window $\left(\mathrm{CD} 3^{-} \mathrm{CD} 4^{+}\right.$cells) divided by the total number of events corresponding to $\mathrm{CD} 4^{+}$cells.

\subsection{Flow cytometry micronucleus test}

Determination of micronuclei (MN) frequency by flow cytometry was performed following the protocol described by Valdiglesias et al. (2011). For each duplicate sample a minimum of 50,000 events were captured in a FACSCalibur flow cytometer (Becton Dickinson) from the propidium iodide (PI) signal detected in the FL2 channel. Data analysis was carried out with Cell Quest Pro software (Becton Dickinson), following the instructions reported by Avlasevich et al. (2006).

\subsection{Comet assay}

After quickly thawing mononuclear leukocytes at $37^{\circ} \mathrm{C}$, cell viability was assessed by trypan blue exclusion technique being, in all cases, higher than $85 \%$. The alkaline comet assay was performed as 
previously described in Laffon et al. (2002), introducing an internal standard following Cebulska-Wasilewska (2003). A single scorer examined 50 randomly selected cells per replicate slide (100 cells/ individual). Comet Assay IV software (Perceptive Instruments) was used for image capture and analysis, considering the percentage of DNA in the comet tail (\%DNAT) as DNA damage parameter.

\subsection{Oxidative damage}

A variant of the comet assay introducing incubation with the repair enzyme OGG1 was used to evaluate the oxidative damage, following the protocol proposed by Smith et al. (2006). Briefly, after lysis the slides were washed 3 times 5 min each with a buffer containing $0.5 \mathrm{mM}$ EDTA, $0.2 \mathrm{mg} / \mathrm{ml} \mathrm{BSA}, 0.1 \mathrm{M} \mathrm{KCl}$ and $40 \mathrm{mM}$ Hepes (pH 8). Fifty microlitres of OGG1 $(0.0016 \mathrm{U} / \mu \mathrm{l}$ buffer $)$ were added to each microgel. Immediately, gels were covered with a coverslip and incubated in a humidified chamber for $10 \mathrm{~min}$ at $37^{\circ} \mathrm{C}$. Four slides were prepared from each individual. Two of them followed the treatment with the enzyme and the other two were incubated only with $50 \mu \mathrm{l}$ of buffer, without enzyme. An internal standard was introduced as described in Cebulska-Wasilewska (2003). After incubation, the coverslips were removed and the slides underwent electrophoresis as described in Laffon et al. (2002). The slides were examined as described in the previous section. The oxidative damage (OGG1-sensitive sites) was calculated as the difference between \%DNAT in the enzymeincubated samples and the \%DNAT in the buffer-incubated samples.

\subsection{Genotyping}

A commercially available kit (NucleoSpin ${ }^{\circledR}$ Blood L, Machery-Nagel) was used to extract genomic DNA from whole blood samples according to the manufacturer's instructions. DNA samples were stored at $-20{ }^{\circ} \mathrm{C}$ until analysis.

\subsubsection{Genes involved in lead toxicokinetics}

The genetic polymorphisms of $A L A D$ (c.177 G > C, p.N59K, rs1800435) and vitamin D receptor (VDR) (c.1024 + $283 \mathrm{G}>\mathrm{A}$, rs1544410) were determined by polymerase chain reaction-restriction fragment length polymorphism (PCR-RFLP) methods described in García-Lestón et al. (2011).

\subsubsection{DNA repair genes}

The XRCC1 (c.580 C > T, p.R194W, rs1799782; c.1196 G > A, p.R399Q rs25487), XRCC2 (c.563 G>A, p.R188H, rs3218536) and XRCC3 (c.722 C > T, p.T241M, rs861539) genetic polymorphisms were analysed by PCR-RFLP as described in Silva et al. (2007) for XRCC1 polymorphisms, and in Bastos et al. (2009) for XRCC2 and XRCC3 polymorphisms.

The following polymorphisms were genotyped by real-time PCR using TaqMan ${ }^{\circledR}$ SNP Genotyping Assays from Applied Biosystems (ABI Assays references are indicated for each polymorphism) following Gomes et al. (2010), Conde et al. (2009) and Silva et al. (2010): XRCC4 (c.8947 G > A, rs1805377, C_11685997_10; c.401 T>C, p.I134T, rs28360135, C_25618660_10), LIG4 (c.26 C > T, p.T9I, rs1805388, C_11427969_20), XRCC5 (c.2199*841 G>A, rs2440, C_3231046_20; c.2199*323 T>C, rs1051677, C_8838367_1; c.2199*451 A > G, rs1051685, C_8838368_1), APE1 (c.444 T > G, p.D148E, rs1130409, C_8921503_10), MUTYH (c.1005 C > G, p.Q335H, rs3219489, C_27504565_10), PARP1 (c.2285 T> C, p.V762A, rs1136410, C_1515368_1), PARP4 (c.3982 C>A, p.P1328T, rs1050112, C_8700142_10), MPG (c.49 A>C, p.K17Q rs3176383, C_ 32323403_10), OGG1 (c.977 C> G, p.S326C, rs1052133, C_3095552_1), RAD51 (c.1-61 G>T, rs1801321, C_7482700_10) and NBS1 (c.553 G>C, p.E185Q rs1805794, C_26470398_10).

\subsection{Statistical analysis}

All analyses were performed using the SPSS for Windows statistical package, version 18.0 (IL, USA). The statistical differences between means and the relationship between categorical variables in the characteristics of the study population were assessed by means of Student's $t$ test and Fisher's exact test or Pearson's Chi-square test, respectively. Analysis of variance (ANOVA) and Bonferroni's test were used to evaluate the existence of differences among groups. The contribution of potential confounding factors to the response variables considered was analysed by multivariate analysis. Associations between variables were assessed by Pearson's correlation. The level of statistical significance was set at 0.05 .

\section{Results}

This study comprised 255 male individuals, 148 lead exposed workers and 107 controls. Results obtained for biomarkers of exposure and genotoxic effect are recorded in Table 2. Regarding the biomarkers of exposure, lead exposed workers showed markedly higher BLL and lower ALAD activity than the controls; this last parameter was significantly lower in workers from factory 1 than in those form factory 2 . In the analysis of genotoxicity biomarkers, significant increases of TCR mutation frequency (TCR-Mf), MN rate and \%DNAT were observed in the exposed group with regard to the controls. Oxidative damage did not experience any significant alteration in the exposed population, but workers from plant 2 presented significantly lower values than those from plant 1.

Multivariant analyses were applied to determine the possible associations between the biomarkers evaluated and lifestyle factors (tobacco consumption and age), and results obtained are summarized in Table 3. Smoking did not influence any variable, and a significant effect of age was only observed on BLL. Indeed, significant correlation was obtained between age and BLL $(r=0.266, P<0.01)$, and also between age and ALAD activity $(r=-0.372, P<0.01)$, TCR-Mf $(r=0.153, P<0.05)$, and MN frequency $(r=0.160$, $P<0.05)$.

In the evaluation of the relationships between the analytical variables assessed, BLL were positively correlated with TCR-Mf $(r=0.381, P<0.01)$, \%NAT $(r=0.230, P<0.01)$ and MN frequency $(r=0.206, P<0.01)$, and inversely correlated with ALAD activity $(\mathrm{r}=-0.491, P<0.01)$. ALAD activity was also inversely correlated with TCR-Mf $(r=$ $-0.191, P<0.05)$ and MN frequency $(r=-0.378, P<0.01)$. Finally, \%DNAT was positively correlated with TCR-Mf $(r=0.295, P<0.01)$.

Twenty single nucleotide polymorphisms were determined in this study; Tables 4 and 5 gather the genotype frequencies in the study populations for the 2 genes involved in the toxicokinetics of lead and for the 14 DNA repair genes, respectively. Significant differences were observed in the genotype distribution of ALAD rs1800435, OGG1 rs1052133 and XRCC5 rs1051677 polymorphisms between exposed population and control group. However, it must be taken into account that for the XRCC5 rs1051677 polymorphism the p value obtained is not accurate since the genotype $\mathrm{C} / \mathrm{C}$ has no representation in the study population. The frequencies of the variant allele in all the polymorphisms were equivalent to those found in the single nucleotide polymorphisms (SNP) database from NCBI (National Center for Biotechnology Information) except for VDR rs1544410 (0.508 vs. 0.267), XRCC1 rs25487 (0.334 vs. 0.266), MUTYH

Table 2

Results obtained in the biomarkers of exposure and effect (mean $\pm \mathrm{SE}$ ).

\begin{tabular}{|c|c|c|c|c|c|c|c|c|}
\hline & $N$ & Controls & $N$ & Exposed & $N$ & Factory 1 & $N$ & Factory 2 \\
\hline BLL $(\mu \mathrm{g} / \mathrm{dl})$ & 107 & $3.65 \pm 0.41$ & 148 & $32.00 \pm 1.09^{* *}$ & 89 & $30.50 \pm 1.22^{*}$ & 59 & $34.26 \pm 1.99^{*}$ \\
\hline ALAD act. (U) & 37 & $91.19 \pm 4.63$ & 124 & $41.40 \pm 2.28^{* *}$ & 79 & $35.58 \pm 2.51^{*}$ & 45 & $51.62 \pm 4.07^{*}, \#$ \\
\hline TCR-Mf & 107 & $6.86 \pm 0.97$ & 91 & $21.06 \pm 1.85^{* *}$ & 49 & $20.76 \pm 2.47^{*}$ & 42 & $21.41 \pm 2.83^{*}$ \\
\hline$\% \mathrm{MN}$ & 107 & $17.80 \pm 0.32$ & 134 & $19.77 \pm 0.28^{* *}$ & 79 & $19.67 \pm 0.30^{*}$ & 55 & $19.92 \pm 0.54^{*}$ \\
\hline \%DNAT & 106 & $3.75 \pm 0.19$ & 138 & $5.72 \pm 1.85^{* *}$ & 82 & $5.69 \pm 0.39^{*}$ & 56 & $5.76 \pm 0.35^{*}$ \\
\hline Ox. damage & 106 & $1.39 \pm 0.24$ & 138 & $1.59 \pm 0.27$ & 82 & $2.10 \pm 0.32$ & 56 & $0.83 \pm 0.44^{\#}$ \\
\hline
\end{tabular}

${ }^{* *} P \leq 0.01,{ }^{*} P \leq 0.05$, significant difference with regard to the control group.

${ }^{\#} P \leq 0.05$, significant difference with regard to factory 1 .

SE: standard error. 
Table 3

Influence of age and smoking habits on biomarkers of exposure and effect. Models adjusted by exposure.

\begin{tabular}{|c|c|c|c|c|c|}
\hline Model & $\begin{array}{l}\text { Unstandardized } \\
\text { coefficients } \beta\end{array}$ & $95 \% \mathrm{CI}$ & $\begin{array}{l}\text { Partial }^{\mathrm{a}} \\
P \text { value }\end{array}$ & $\mathrm{R}^{2}$ & $\begin{array}{l}\text { Model }^{\mathrm{b}} \\
P \text { value }\end{array}$ \\
\hline \multicolumn{6}{|l|}{ 1. BLL ( $\mu \mathrm{g} / \mathrm{dl})$} \\
\hline Age (years) & 0.154 & 0.032 to 0.276 & 0.014 & 0.653 & $<0.001$ \\
\hline $\begin{array}{l}\text { Smokers vs. } \\
\text { non-smokers }\end{array}$ & 0.420 & -2.474 to 3.314 & 0.775 & 0.644 & $<0.001$ \\
\hline \multicolumn{6}{|l|}{ 2. ALAD act. (U) } \\
\hline Age (years) & -0.140 & -0.606 to 0.327 & 0.555 & 0.398 & $<0.001$ \\
\hline $\begin{array}{l}\text { Smokers vs. } \\
\text { non-smokers }\end{array}$ & 1.044 & -7.598 to 9.686 & 0.812 & 0.397 & $<0.001$ \\
\hline \multicolumn{6}{|l|}{ 3. TCR-Mf } \\
\hline Age (years) & 0.078 & -0.106 to 0.263 & 0.404 & 0.206 & $<0.001$ \\
\hline $\begin{array}{l}\text { Smokers vs. } \\
\text { non-smokers }\end{array}$ & 4.000 & -0.550 to 8.550 & 0.085 & 0.215 & $<0.001$ \\
\hline \multicolumn{6}{|l|}{ 4. \%MN } \\
\hline Age (years) & 0.032 & -0.008 to 0.072 & 0.112 & 0.093 & $<0.001$ \\
\hline $\begin{array}{l}\text { Smokers vs. } \\
\text { non-smokers }\end{array}$ & 0.573 & -0.359 to 1.505 & 0.227 & 0.088 & $<0.001$ \\
\hline \multicolumn{6}{|l|}{ 5. \%DNAT } \\
\hline Age (years) & 0.007 & -0.027 to 0.040 & 0.693 & 0.115 & $<0.001$ \\
\hline $\begin{array}{l}\text { Smokers vs. } \\
\text { non-smokers }\end{array}$ & 0.370 & -0.404 to 1.145 & 0.347 & 0.118 & $<0.001$ \\
\hline \multicolumn{6}{|l|}{ 5. Ox. damage } \\
\hline Age (years) & -0.016 & -0.051 to 0.019 & 0.394 & 0.005 & 0.578 \\
\hline $\begin{array}{l}\text { Smokers vs. } \\
\text { non-smokers }\end{array}$ & 0.417 & -0.400 to 1.235 & 0.316 & 0.005 & 0.528 \\
\hline
\end{tabular}

a Significance of the particular variable (age or smoking) on the response variable.

b Significance of the model applied.

rs3219489 (0.276 vs, 0.319), PARP1 rs1136410 (0.148 vs. 0.244), XRCC2 rs3218536 (0.118 vs. 0.043), XRCC3 rs861539 (0.448 vs. 0.251), RAD51 rs1801321 (0.503 vs. $0.265)$, XRCC4 rs28360135 (0.054 vs. 0.016), XRCC4 rs1805377 (0.136 vs. 0.393$)$ and XRCC5 rs 1051677 (0.089 vs. 0.125).

The possible influence of the polymorphisms in lead toxicokinetics genes was investigated in both exposure and genotoxic effect biomarkers; for DNA repair genes, this influence was only assessed in genotoxicity biomarkers. All the exposed and control individuals were wild-type homozygous for MPG rs3176383 polymorphism, so it could not be included in the evaluation. Due to the large amount of data generated in these analyses, only statistically significant results are shown in Table 6. None of the exposure or genotoxicity biomarkers were significantly influenced by polymorphisms in ALAD, XRCC1, APE1, PARP1, XRCC5, XRCC2, XRCC3, RAD51 or NBS genes. VDR rs1544410 polymorphism influenced BLL, since carriers of the variant allele B showed lower BLL than wild-type $\mathrm{b}$ homozygotes, although significance was only reached for $\mathrm{B} / \mathrm{B}$ homozygotes. The presence of the $\mathrm{G}$ variant allele of APE1 rs1130409 was associated with an increase in TCR-Mf, significant only in G/G homozygous individuals. LIG4 rs1805388 polymorphism influenced both TCR-Mf and \%DNAT, significantly only in $\mathrm{T} / \mathrm{T}$ homozygotes, which showed higher values of the genotoxicity parameters than wild-type $\mathrm{C} / \mathrm{C}$ homozygous individuals. Individuals who carry the MUTYH rs3219489 $\mathrm{G}$ variant allele displayed higher levels of DNA damage than wild-type C/C homozygotes; the difference was significant for the comparison with heterozygous subjects. The same influence on DNA damage was observed in XRCC4 rs28360135 C variant allele carriers, although in this case the significance was obtained in $\mathrm{C} / \mathrm{C}$ homozygous individuals. Besides, XRCC4 rs28360135 polymorphism was also associated with a decrease in oxidative damage in C allele carriers, significant only in homozygous individuals. Oxidative damage was also influenced by OGG1 rs1052133, as individuals carrying $\mathrm{G}$ variant allele presented higher damage than $\mathrm{C} / \mathrm{C}$ homozygotes; significance was reached only in $\mathrm{G} / \mathrm{G}$ homozygous individuals.

Table 4

Genotype distribution of genes involved in lead toxicokinetics in control and exposed groups.

\begin{tabular}{lllll}
\hline SNP & Genotype & Controls $N(\%)$ & Exposed $N(\%)$ & $P$ value $^{\mathrm{a}}$ \\
\hline VDR rs1544410 & $\mathrm{b} / \mathrm{b}$ & $33(32.0)$ & $40(27.0)$ & 0.540 \\
& $\mathrm{~B} / \mathrm{b}$ & $42(40.8)$ & $59(39.9)$ & \\
\multicolumn{6}{l}{ Variant allele frequency } & $\mathrm{B} / \mathrm{B}$ & 0.476 & $49(33.1)$ & \\
ALAD rs1800435 & $1 / 1$ & $85(86.7)$ & 0.531 & 0.003 \\
& $1 / 2$ & $13(13.3)$ & $3(2.3)$ & \\
\multicolumn{7}{l}{ Variant allele frequency } & $2 / 2$ & 0 & $2(1.5)$ & \\
\hline
\end{tabular}

\footnotetext{
a Pearson's Chi-square (two tail).
}

Table 5

Genotype distribution of DNA repair genes in control and exposed groups.

\begin{tabular}{|c|c|c|c|c|c|}
\hline $\begin{array}{l}\text { Repair } \\
\text { pathway }\end{array}$ & SNP & Genotype & $\begin{array}{l}\text { Controls } \\
N(\%)\end{array}$ & $\begin{array}{l}\text { Exposed } \\
N(\%)\end{array}$ & $P$ value $^{\mathrm{a}}$ \\
\hline \multirow[t]{3}{*}{ BER } & XRCC1 rs1799782 & $\mathrm{C} / \mathrm{C}$ & $70(84.3)$ & $116(78.4)$ & 0.540 \\
\hline & & $\mathrm{C} / \mathrm{T}$ & $12(14.5)$ & $29(19.6)$ & \\
\hline & & $\mathrm{T} / \mathrm{T}$ & $1(1.2)$ & $3(2)$ & \\
\hline \multicolumn{2}{|c|}{ Variant allele frequency } & & 0.085 & 0.118 & \\
\hline \multirow{3}{*}{\multicolumn{2}{|c|}{ XRCC1 rs25487 }} & $\mathrm{G} / \mathrm{G}$ & $\overline{35(42.2)}$ & $\overline{68(45.9)}$ & 0.728 \\
\hline & & $\mathrm{G} / \mathrm{A}$ & $37(44.6)$ & 65 (43.9) & \\
\hline & & $\mathrm{A} / \mathrm{A}$ & $11(13.3)$ & $15(10.1)$ & \\
\hline \multicolumn{2}{|c|}{ Variant allele frequency } & & 0.356 & 0.321 & \\
\hline \multirow{3}{*}{\multicolumn{2}{|c|}{ APE1 rs1130409 }} & $T / T$ & $\overline{27(32.5)}$ & $\overline{40(27.2)}$ & 0.251 \\
\hline & & $\mathrm{T} / \mathrm{G}$ & $37(44.6)$ & $82(55.8)$ & \\
\hline & & $\mathrm{G} / \mathrm{G}$ & $19(22.9)$ & $25(17)$ & \\
\hline \multicolumn{2}{|c|}{ Variant allele frequency } & & 0.452 & 0.449 & \\
\hline \multirow{3}{*}{\multicolumn{2}{|c|}{ MUTYH rs3219489 }} & $\mathrm{C} / \mathrm{C}$ & $\overline{44(53)}$ & $\overline{80(54.1)}$ & 0.491 \\
\hline & & $\mathrm{C} / \mathrm{G}$ & $34(41)$ & $53(35.8)$ & \\
\hline & & $\mathrm{G} / \mathrm{G}$ & $5(6)$ & $15(10.1)$ & \\
\hline \multicolumn{3}{|c|}{ Variant allele frequency } & 0.265 & 0.280 & \\
\hline \multirow{3}{*}{\multicolumn{2}{|c|}{ PARP1 rs 1136410}} & $\mathrm{~T} / \mathrm{T}$ & $\overline{55(66.3)}$ & $\overline{110(75.3)}$ & 0.327 \\
\hline & & $\mathrm{T} / \mathrm{C}$ & $26(31.3)$ & $34(23.3)$ & \\
\hline & & $\mathrm{C} / \mathrm{C}$ & $2(2.4)$ & $2(1.4)$ & \\
\hline \multicolumn{2}{|c|}{ Variant allele frequency } & & 0.181 & 0.131 & \\
\hline & PARP4 rs1050112 & $\mathrm{C} / \mathrm{C}$ & $\overline{30(36.1)}$ & $\overline{62(42.2)}$ & 0.335 \\
\hline & & $\mathrm{C} / \mathrm{A}$ & $40(48.2)$ & $71(48.3)$ & \\
\hline & & $\mathrm{A} / \mathrm{A}$ & $13(15.7)$ & $14(9.5)$ & \\
\hline Variant al & ele frequency & & 0.398 & 0.337 & \\
\hline & MPG rs3176383 & $\mathrm{A} / \mathrm{A}$ & $\overline{83(100)}$ & $\overline{146(100)}$ & \\
\hline & & $\mathrm{A} / \mathrm{C}$ & 0 & 0 & \\
\hline & & $\mathrm{C} / \mathrm{C}$ & 0 & 0 & \\
\hline Variant al & ele frequency & & 0 & 0 & \\
\hline & OGG1 rs1052133 & $\mathrm{C} / \mathrm{C}$ & $\overline{5} 5(66.3)$ & $\overline{8} 3(56.5)$ & 0.036 \\
\hline & & $\mathrm{C} / \mathrm{G}$ & $28(33.7)$ & $54(36.7)$ & \\
\hline & & $\mathrm{G} / \mathrm{G}$ & 0 & $10(6.8)$ & \\
\hline Variant al & ele frequency & & 0.169 & 0.252 & \\
\hline NHEJ & XRCC4 rs28360135 & $\mathrm{T} / \mathrm{T}$ & $\overline{75(90.4)}$ & $\overline{132(89.2)}$ & 0.747 \\
\hline & & $\mathrm{T} / \mathrm{C}$ & $8(9.6)$ & $15(10.1)$ & \\
\hline & & $\mathrm{C} / \mathrm{C}$ & 0 & $1(0.7)$ & \\
\hline Variant al & ele frequency & & 0.048 & 0.575 & \\
\hline & XRCC4 rs1805377 & $\mathrm{G} / \mathrm{G}$ & $\overline{67(80.7)}$ & $\overline{108(74)}$ & 0.086 \\
\hline & & $\mathrm{G} / \mathrm{A}$ & $16(19.3)$ & $30(20.5)$ & \\
\hline & & $\mathrm{A} / \mathrm{A}$ & 0 & $8(5.5)$ & \\
\hline Variant al & ele frequency & & 0.097 & 0.158 & \\
\hline & LIG4 rs1805388 & $\mathrm{C} / \mathrm{C}$ & $\overline{64(77.1)}$ & $\overline{113(76.4)}$ & 0.964 \\
\hline & & $\mathrm{C} / \mathrm{T}$ & $17(20.5)$ & $32(21.6)$ & \\
\hline & & $\mathrm{T} / \mathrm{T}$ & $2(2.4)$ & $3(2)$ & \\
\hline Variant al & ele frequency & & 0.127 & 0.128 & \\
\hline & XRCC5 rs2440 & $\mathrm{G} / \mathrm{G}$ & $\overline{26(18.1)}$ & $\overline{35(23.6)}$ & 0.415 \\
\hline & & $\mathrm{G} / \mathrm{A}$ & $41(49.4)$ & $78(52.7)$ & \\
\hline & & $\mathrm{A} / \mathrm{A}$ & $16(32.5)$ & $35(23.6)$ & \\
\hline Variant al & ele frequency & & 0.572 & 0.500 & \\
\hline & XRCC5 rs1051677 & $\mathrm{T} / \mathrm{T}$ & $\overline{76(91.6)}$ & $\overline{114(77)}$ & 0.006 \\
\hline & & $\mathrm{T} / \mathrm{C}$ & $7(8.4)$ & $34(23)$ & \\
\hline & & $\mathrm{C} / \mathrm{C}$ & 0 & 0 & \\
\hline Variant al & ele frequency & & 0.042 & 0.115 & \\
\hline & XRCC5 rs1051685 & $\mathrm{A} / \mathrm{A}$ & $\overline{64(77.1)}$ & $\overline{120(81.8)}$ & 0.745 \\
\hline & & $\mathrm{A} / \mathrm{G}$ & $18(21.7)$ & $26(16.9)$ & \\
\hline & & $\mathrm{G} / \mathrm{G}$ & $1(1.2)$ & $2(1.4)$ & \\
\hline Variant al & ele frequency & & 0.121 & 0.100 & \\
\hline $\mathrm{HR}$ & XRCC2 rs3218536 & $\mathrm{G} / \mathrm{G}$ & $\overline{66(15.7)}$ & $\overline{128(86.5)}$ & 0.199 \\
\hline & & $\mathrm{G} / \mathrm{A}$ & $16(19.3)$ & $20(13.5)$ & \\
\hline & & $\mathrm{A} / \mathrm{A}$ & $1(65.1)$ & 0 & \\
\hline Variant al & ele frequency & & 0.254 & 0.068 & \\
\hline & XRCC3 rs861539 & $\mathrm{C} / \mathrm{C}$ & $\overline{24(28.9)}$ & $\overline{49(33.3)}$ & 0.770 \\
\hline & & $\mathrm{C} / \mathrm{T}$ & $40(48.2)$ & $68(46.3)$ & \\
\hline & & $\mathrm{T} / \mathrm{T}$ & $19(22.9)$ & $30(20.4)$ & \\
\hline Variant al & ele frequency & & 0.470 & 0.436 & \\
\hline & RAD51 rs1801321 & $\mathrm{G} / \mathrm{G}$ & $\overline{26(26.5)}$ & $\overline{36(23.6)}$ & 0.473 \\
\hline & & $\mathrm{G} / \mathrm{T}$ & $37(44.6)$ & $69(46.6)$ & \\
\hline & & $\mathrm{T} / \mathrm{T}$ & $20(28.9)$ & $43(29.7)$ & \\
\hline Variant al & ele frequency & & 0.512 & 0.530 & \\
\hline & NBS1 rs1805794 & $\mathrm{G} / \mathrm{G}$ & $\overline{36(43.4)}$ & $\overline{67(45.3)}$ & 0.895 \\
\hline & & $\mathrm{G} / \mathrm{C}$ & $37(44.6)$ & $66(44.6)$ & \\
\hline & & $\mathrm{C} / \mathrm{C}$ & $10(12)$ & $15(10.1)$ & \\
\hline Variant al & ele frequency & & 0.343 & 0.324 & \\
\hline
\end{tabular}

a Pearson's Chi-square (two tail). 
Table 6

Biomarkers of susceptibility with significant influence on exposure or genotoxicity biomarkers. Models adjusted by exposure, age and tobacco consumption.

\begin{tabular}{|c|c|c|c|c|c|c|}
\hline Model & Genotype & $\begin{array}{l}\text { Unstandardized } \\
\text { coefficients } \beta\end{array}$ & $95 \% \mathrm{CI}$ & $\begin{array}{l}\text { Partial }^{\mathrm{a}} \\
P \text { value }\end{array}$ & $\mathrm{R}^{2}$ & $\begin{array}{l}\text { Model }^{\mathrm{b}} \\
P \text { value }\end{array}$ \\
\hline \multicolumn{7}{|l|}{ 1. BLL $(\mu \mathrm{g} / \mathrm{dl})$} \\
\hline VDR rs1544410 & $\begin{array}{l}\mathrm{B} / \mathrm{b} v s . \mathrm{b} / \mathrm{b} \\
\mathrm{B} / \mathrm{B} v s . \mathrm{b} / \mathrm{b}\end{array}$ & $\begin{array}{l}-0.683 \\
-4.444\end{array}$ & $\begin{array}{l}-3.801 \text { to } 2.435 \\
-7.763 \text { to }-1.125\end{array}$ & $\begin{array}{l}0.667 \\
0.009\end{array}$ & 0.662 & $<0.001$ \\
\hline \multicolumn{7}{|l|}{ 2. TCR-Mf } \\
\hline APE1 rs1130409 & $\begin{array}{l}\mathrm{T} / \mathrm{G} v s . \mathrm{T} / \mathrm{T} \\
\mathrm{G} / \mathrm{G} v s . \mathrm{T} / \mathrm{T}\end{array}$ & $\begin{array}{l}1.135 \\
7.864\end{array}$ & $\begin{array}{l}-3.896 \text { to } 6.167 \\
1.314 \text { to } 14.414\end{array}$ & $\begin{array}{l}0.657 \\
0.019\end{array}$ & 0.237 & $<0.001$ \\
\hline LIG4 rs1805388 & $\begin{array}{l}\mathrm{C} / \mathrm{T} \text { vs. } \mathrm{C} / \mathrm{C} \\
\mathrm{T} / \mathrm{T} \text { vs. } \mathrm{C} / \mathrm{C}\end{array}$ & $\begin{array}{l}4.603 \\
20.787\end{array}$ & $\begin{array}{l}-0.929 \text { to } 10.136 \\
7.867 \text { to } 33.707\end{array}$ & $\begin{array}{l}0.102 \\
0.002\end{array}$ & 0.259 & $<0.001$ \\
\hline \multicolumn{7}{|l|}{ 3. \%DNAT } \\
\hline MUTYH rs3219489 & $\begin{array}{l}\mathrm{C} / \mathrm{G} \text { vs. } \mathrm{C} / \mathrm{C} \\
\mathrm{G} / \mathrm{G} \text { vs. } \mathrm{C} / \mathrm{C}\end{array}$ & $\begin{array}{l}1.255 \\
1.103\end{array}$ & $\begin{array}{l}0.467 \text { to } 2.044 \\
-0.273 \text { to } 2.480\end{array}$ & $\begin{array}{l}0.002 \\
0.115\end{array}$ & 0.160 & $<0.001$ \\
\hline XRCC4 rs28360135 & $\begin{array}{l}\mathrm{C} / \mathrm{T} \text { vs. } \mathrm{T} / \mathrm{T} \\
\mathrm{C} / \mathrm{C} \text { vs. T/T }\end{array}$ & $\begin{array}{l}0.588 \\
7.346\end{array}$ & $\begin{array}{l}-0.643 \text { to } 1.819 \\
1.852 \text { to } 12.839\end{array}$ & $\begin{array}{l}0.348 \\
0.009\end{array}$ & 0.149 & $<0.001$ \\
\hline LIG4 rs1805388 & $\begin{array}{l}\mathrm{C} / \mathrm{T} \text { vs. } \mathrm{C} / \mathrm{C} \\
\mathrm{T} / \mathrm{T} \text { vs. } \mathrm{C} / \mathrm{C}\end{array}$ & $\begin{array}{l}-0.530 \\
3.155\end{array}$ & $\begin{array}{l}-1.444 \text { to } 0.385 \\
0.674 \text { to } 5.637\end{array}$ & $\begin{array}{l}0.255 \\
0.013\end{array}$ & 0.151 & $<0.001$ \\
\hline 4. Ox. damage & & & & & & \\
\hline OGG1 rs1052133 & $\begin{array}{l}\mathrm{C} / \mathrm{G} \text { vs. } \mathrm{C} / \mathrm{C} \\
\mathrm{G} / \mathrm{G} \text { vs. } \mathrm{C} / \mathrm{C}\end{array}$ & $\begin{array}{l}0.123 \\
3.379\end{array}$ & $\begin{array}{l}-0.692 \text { to } 0.938 \\
1.479 \text { to } 5.279\end{array}$ & $\begin{array}{l}0.766 \\
0.001\end{array}$ & 0.065 & 0.014 \\
\hline XRCC4 rs28360135 & $\begin{array}{l}\mathrm{C} / \mathrm{T} \text { vs. T/T } \\
\mathrm{C} / \mathrm{C} \text { vs. T/T }\end{array}$ & $\begin{array}{l}-0.830 \\
-11.743\end{array}$ & $\begin{array}{l}-2.094 \text { to } 0.434 \\
-17.383 \text { to }-6.103\end{array}$ & $\begin{array}{l}0.197 \\
0.000\end{array}$ & 0.088 & 0.001 \\
\hline
\end{tabular}

a Significance of the particular comparison between genotypes.

b Significance of the model applied.

Finally, as some genotoxicity parameters were significantly influenced by more than one genetic polymorphism, we calculated a categorical variable by grouping the subjects according to the number of variant alleles in each group of genes that showed a significant influence on each genotoxicity parameter, in order to test if they may have a combined effect, and the results are gathered in Table 7. The increase in the number of variant alleles of APE1 rs1130409 and LIG4 rs1805388 polymorphisms was associated with an increase in TCR-Mf, significant in the group of individuals with 3 or more variant alleles. \%DNAT also increased with the number of variant alleles of MUTYH rs3219489, XRCC4 rs28360135 and LIG4 rs1805388 polymorphisms; significance was reached in all subjects with more than 1 variant allele. Besides, an increase in the oxidative damage was observed in individuals carrying 2 variant alleles of OGG1 rs1052133, and XRCC4 rs28360135 polymorphisms, but in this case the model did not reach the statistical significance.

\section{Discussion}

In this study we evaluated the genotoxic effects of lead exposure in a population of 148 workers from two Portuguese factories, and compared them with 107 controls. Lead exposure was assessed by means of BLL and ALAD activity, since they are the most widely used and reliable biomarkers of lead exposure (Sakai, 2000). BLL showed a 9-fold increase in the exposed workers with regard to the controls. Although none of the exposed individuals exceeded the $70 \mu \mathrm{g} / \mathrm{dl}$ limit established by the Portuguese regulation, 47 exposed workers (32\%) went over the US Occupational Safety and Health Administration (OSHA) limit $(40 \mu \mathrm{g} / \mathrm{dl})$. Contrary to BLL, and according to the strong ALAD inhibitory role of lead, a significant decrease in ALAD activity was observed in the exposed group when compared to the controls; indeed, ALAD activity and BLL were negatively correlated. Moreover, workers from factory 2 showed significantly higher ALAD activity than those from factory 1 , probably due to the different activities developed in the two factories.

We did not find relationship between BLL and time working in a lead contaminated environment. Indeed, BLL are not cumulative; they reflect the exposure occurred only during the last weeks (Schwartz et al., 1994). However, BLL were significantly and positively influenced by age, suggesting modifications in lead toxicokinetics that entail higher BLL with increasing age under a given exposure level.

In order to evaluate comprehensively the possible genotoxic effects of occupational exposure to lead, we analysed four different genotoxicity biomarkers (TCR mutation assay, MN test, comet assay, and oxidative damage as OGG1 sensitive sites in the comet assay), since they can reflect different mechanisms of damage induction. Our data showed significant increases in TCR-Mf, MN frequency and primary DNA damage in the lead exposed workers, confirming the genotoxicity associated with this occupational exposure, not only in the short term (comet assay results) but also in the medium-long term (TCR mutation assay and MN test). Moreover, all these 3 parameters were significantly correlated with BLL. These results generally agree with most studies that evaluated genotoxicity parameters (comet assay, MN test, chromosomal aberrations) in people occupationally exposed to lead in different occupational settings with BLL similar to the ones in

Table 7

Influence of the number of variant alleles of DNA repair genes on genotoxicity biomarkers. Models adjusted by exposure, age and tobacco consumption.

\begin{tabular}{|c|c|c|c|c|c|c|}
\hline Model & $\begin{array}{l}\text { No. of variant } \\
\text { alleles }(N)\end{array}$ & $\begin{array}{l}\text { Unstandardized } \\
\text { coefficients } \beta\end{array}$ & $95 \% \mathrm{CI}$ & $\begin{array}{l}\text { Partial }^{\mathrm{a}} \\
P \text { value }\end{array}$ & $\mathrm{R}^{2}$ & $\begin{array}{l}\text { Model }^{\mathrm{b}} \\
P \text { value }\end{array}$ \\
\hline \multicolumn{7}{|l|}{ 1. TCR-Mf } \\
\hline \multirow[t]{3}{*}{ APE1 rs1130409 and LIG4 rs1805388 } & 1 (79) vs. 0 (43) & 0.686 & -4.632 to 6.01 & 0.799 & \multirow[t]{3}{*}{0.284} & \multirow[t]{3}{*}{$<0.001$} \\
\hline & 2 (40) vs. $0(43)$ & 4.479 & -1.746 to 10.71 & 0.157 & & \\
\hline & $\geq 3$ (11) vs. 0 (43) & 18.535 & 9.07 to 28.00 & 0.000 & & \\
\hline \multicolumn{7}{|l|}{ 2. \%DNAT } \\
\hline \multirow[t]{3}{*}{ MUTYH rs3219489, XRCC4 rs28360135 and LIG4 rs1805388 } & 1 (97) vs. 0 (79) & 0.400 & -0.420 to 1.220 & 0.338 & \multirow[t]{3}{*}{0.149} & \multirow[t]{3}{*}{$<0.001$} \\
\hline & 2 (33) vs. 0 (79) & 1.184 & 0.063 to 2.305 & 0.038 & & \\
\hline & $\geq 3$ (10) vs. 0 (79) & 1.999 & 0.182 to 3.817 & 0.031 & & \\
\hline \multicolumn{7}{|l|}{ 3. Ox. damage } \\
\hline \multirow[t]{2}{*}{ OGG1 rs1052133 and XRCC4 rs28360135 } & 1 (89) vs. 0 (113) & -0.196 & 0.989 to 0.597 & 0.627 & \multirow[t]{2}{*}{0.041} & \multirow[t]{2}{*}{0.113} \\
\hline & 2 (16) vs. 0 (113) & 1.819 & 0.313 to 3.324 & 0.018 & & \\
\hline
\end{tabular}

\footnotetext{
a Significance of the particular comparison between genotypes.
}

b Significance of the model applied. 
our population (Grover et al., 2010; Kasuba et al., 2010; Khan et al., 2010; and previous works reviewed in García-Lestón et al., 2010). Thus, all these data indicate that lead exposure in the workplace induces mutagenicity, chromosomal alterations and DNA damage (strand breaks and/or alkali-labile sites). In this study the origin of the MN observed was not investigated, but results from Palus et al. (2003), who combined MN test with fluorescence in situ hybridization (FISH) with pancentromeric probes, showed that the increase in the MN frequency in workers from a battery plant, mainly exposed to lead, was due to clastogenic as well as aneugenic effects. The negative results obtained in our previous study regarding comet assay (García-Lestón et al., 2011), and in the only previous study which evaluated TCR-Mf in a population of lead exposed workers (Chen et al., 2006), are probably explained by the lower sample sizes (70 and 25 exposed individuals, respectively). As far as we know, the current study comprises the largest group of lead exposed workers analysed for genotoxicity.

Nevertheless, we did not observe differences in the oxidative DNA damage between exposed and control individuals. Previous studies that assessed oxidative stress in workers exposed to lead reported positive results (Ergurhan-Ilhan et al., 2008; Gurer-Orhan et al., 2004; Ye et al., 1999). Parameters analysed in these studies were concentration of malondialdehyde (MDA), alpha-tocopherol, beta-carotene, glutathione:glutathione disulfide ratio, and activity of enzymes like superoxide dismutase (SOD), glucose-6-phosphate dehydrogenase (G6PD), catalase and glutathione peroxidase (GPx). Incorporating incubation with OGG1 in the comet assay, we determined the presence of 8-oxo-7,8dihydroguanine (8-oxoGua), the main result of oxidative DNA damage (Angerer et al., 2007) and one of the most abundant and highly mutagenic base lesions (Laantri et al., 2011). Therefore, it seems that the oxidative stress induced by lead exposure causes lipid peroxidation, depletion of antioxidants, and increase in antioxidant enzyme activities but, according to our results, it does not affect the genetic material. Nevertheless, despite no significant differences observed in the exposed workers regarding to controls, our results showed a significant difference in oxidative DNA damage between the two plants, being higher in plant 1 regarding to plant 2 . This suggests that the type of activity developed in plant 1 involves, in addition to larger decrease in ALAD activity, higher risk of induction of oxidative DNA damage.

Although the influence of age on both TCR-Mf and MN frequency was previously reported and even quantified (Akiyama et al., 1995; Bonassi et al., 2001), our results of the multivariate analysis did not show any significant influence for this factor even though the age range covered by our study group was wide (21-67). Nevertheless, slight but significant positive correlations were obtained between age and the two genotoxicity parameters.

Vitamin D receptor (VDR) plays a major role in maintaining calcium and phosphate homeostasis, in regulating bone metabolism, and is also involved in anti-proliferative, pro-apoptotic and immunosuppressive activities (Hendy et al., 2006). Schwartz et al. (2000) reported that VDR B allele carriers for the rs 1544410 polymorphism have higher BLL and bone lead content than subjects with bb genotype. On the contrary, our results showed significantly lower BLL in BB individuals. However, Schwartz et al. analyzed together all individuals carrying $B$ allele $(\mathrm{bB}+\mathrm{BB})$, and they included among the lead exposed workers an undetermined number of retired workers, who are supposed to have ceased their exposure to lead some time ago. These facts might help to explain these different results. Besides, linkage disequilibrium of this probably non-functional VDR polymorphism (it is located in intron 8) with one or more truly functional polymorphisms elsewhere in the VDR gene was suggested (Uitterlinden et al., 2004).

The human APE1 gene encodes the major apurinic/apyrimidinic (AP) endonuclease in mammalian cells (APE1) that plays a central role in BER pathway by hydrolyzing the phosphodiester backbone immediately $5^{\prime}$ to AP sites in DNA. These AP sites constitute cytotoxic and mutagenic lesions that can prevent normal DNA replication if unrepaired (Cao et al., 2011). Two studies using X-ray crystallography and nuclear magnetic resonance spectroscopy observed that lead may occupy two potential divalent metal binding sites within the APE1 active site, disrupting the magnesium-dependent catalytic reaction that is essential for enzymatic activity (Beernink et al., 2001; Lowry et al., 2003). Moreover, McNeill et al. (2004) reported that lead inhibits APE1 incision activity in vitro, and thus suggested that lead can elicit its mutagenic effects in part by inactivating APE1 repair activities. Although APE1 rs1130409 polymorphism has no impact on DNA binding capacity or endonuclease activity of the protein (Hadi et al., 2000), the overall results of a recent meta-analysis performed by Gu et al. (2009) indicated that the variant APE1 rs1130409 genotypes were associated with a moderately increased risk of all cancer types. Similarly, in the current study we observed higher levels of TCR-Mf in APE1 rs1130409 $\mathrm{G}$ allele carriers. As a hypothesis, maybe lead substitutes magnesium more efficiently in the protein encoded by this allele, so it is more susceptible to be inhibited by lead in exposed individuals.

The human MUTYH gene encodes a DNA glycosylase that excises adenine bases from DNA backbone at sites where adenine is inappropriately paired with guanine, cytosine, or 8-oxoGua (Wilson et al., 2011). Our results showed higher levels of DNA damage related to the presence of MUTYH rs3219489 variant allele G, what is in agreement with a study done with human lymphocytes exposed to glycidamide (Pingarilho et al., submitted). However, in our study the higher levels of DNA damage were only obtained for the C/G heterozygotes, possibly due the small representation of the $G / G$ homozygous genotype in the whole population. In other studies, no association between MUTYH rs3219489 polymorphism and breast cancer risk (Conde et al., 2009) or non-small cell lung cancer risk (Qian et al., 2010) was reported.

The human 8-oxo-guanine glycosylase 1 (OGG1) gene encodes a protein responsible for the cleavage of 8-oxoGua, a major form of base lesion produced by oxidative damage to DNA. If this lesion is not excised, it can be matched with adenine leading to a GC to TA transversion. Therefore, decreased function of OGG1 could lead to mutations that activate oncogenes or inactive tumour suppressor genes (Laantri et al., 2011; Roberts et al., 2011). The OGG1 rs1052133 variant protein has been reported to have a lower ability to prevent mutagenesis (Yamane et al., 2004), supporting the results of association studies that $\mathrm{G}$ is a risk allele for several types of human cancer, including prostate (Chen et al., 2003), lung (Kohno et al., 2006), gastric (Farinati et al., 2008), and head and neck (Kumar et al., 2011). On the contrary, other studies did not find any association between this polymorphism and risk of breast cancer (Roberts et al., 2011), thyroid cancer (GarcíaQuispes et al., 2011) or nasopharyngeal cancer (Laantri et al., 2011).

Results of our study showed a significant increase of oxidative damage in homozygous individuals for the OGG1 rs1052133 G variant allele, suggesting a less effective repair of 8-oxoGua DNA lesions. According to this, Hill and Evans (2006) reported altered substrate specificity, lack of stimulation by APE1 and anomalous DNA binding conformation for the variant enzyme OGG1. Moreover, Bravard et al. (2009) observed that cells homozygous for the G variant allele display reduced in vivo 8-oxoGua repair rates, and also their OGG1 activity is more sensitive to inactivation by oxidizing agents, suggesting that homozygous individuals for the variant allele could accumulate more mutations under conditions of oxidative stress.

XRCC4 and LIG4 genes encode two proteins that form a complex involved in the alignment and rejoining of DNA ends in the final step of NHEJ, the major pathway used by human cells to repair DNA double strand breaks (Wu et al., 2009). Any amino acid exchange that results in diminished stability of this complex could result in a lower activity and consequently a defect in the DNA repair (Gomes et al., 2010). Previous studies investigating the association between XRCC4 or LIG4 polymorphisms and risk of different cancer types reported negative (Gomes et al., 2010; Roddam et al., 2002) and positive (Figueroa et al., 2007; Tseng et al., 2009; Werbrouck et al., 2008) results. In the current work homozygous variant allele carriers of LIG4 rs1805388 (T/T) or XRCC4 rs28360135 (C/C) polymorphisms showed higher values of 
TCR-Mf (LIG4 rs1805388 polymorphism) and DNA damage (both polymorphisms), suggesting a possible relationship between the presence of the variant alleles and a less effective DNA repair activity. Nevertheless, the presence of the XRCC4 rs28360135 C variant allele was associated with a lower level of oxidative DNA damage. The meaning of this result is somewhat conflicting, since the main oxidative DNA lesion detected (8-oxoGua) is preferentially repaired by the BER pathway and not by NHEJ (Robertson et al., 2009).

Apart from the differences observed in the levels of TCR-Mf, \%DNAT and oxidative damage related to single DNA repair gene polymorphisms, we also found that the increasing number of variant alleles of APE1 rs1130409 and LIG4 rs1805388 polymorphisms was associated with an increase in TCR-Mf, and the same occurred for MUTYH rs3219489, XRCC4 rs28360135 and LIG4 rs1805388 polymorphisms with regard to \%TDNA. The simultaneous involvement of two or more polymorphic genes in the levels of genotoxic damage denotes the importance of the simultaneous evaluation of multiple polymorphic genes related with DNA repair processes for a more precise assessment of the individual susceptibility to different types of DNA lesions. However, the combined effect of OGG1 rs1052133 and XRCC4 rs28360135 polymorphisms obtained on oxidative damage has no plausibility, since the effect of each single variant allele on the genotoxicity parameter was opposite, and also the multivariate model was not significant.

In summary, these results showed genotoxic effects related to occupational lead exposure to levels under the limit of $70 \mu \mathrm{g} / \mathrm{dl}$ established by the Portuguese regulation, confirming our previous results (GarcíaLestón et al., 2011). Therefore, we reaffirm the recommendation of reducing the threshold limit for BLL in the Portuguese regulation in order to preserve workers' safety; a limit $<20 \mu \mathrm{g} / \mathrm{dl}$, even lower than OSHA limit ( $40 \mu \mathrm{g} / \mathrm{dl}$ ), was already indicated by Schwartz and Hu (2007). Besides, significant influences of VDR gene polymorphism on BLL, and of polymorphisms in genes involved in BER and NHEJ, but not in HR, on genotoxicity biomarkers were observed, suggesting a role for DNA repair gene polymorphisms as susceptibility biomarkers in occupational genotoxicity biomonitoring.

\section{Acknowledgements}

This work was partly supported by the Spanish Ministry of Science and Innovation (PSI2010-15115) and Portuguese Fundação para a Ciência e a Tecnologia (grants PDCT/SAU-OBS/59821/2004, PTDC/QUI/ 67522/2006 and PTDC/SAU-OSM/105572/2008, and fellowship SFRH/ BD/22612/2005 to M. Pingarilho).

\section{References}

Akiyama M, Umeki S, Kusunoki Y, Kyoizumi S, Nakamura N, Mori T, et al. Somatic-cell mutations as a possible predictor of cancer risk. Health Phys 1995;68:643-9.

Angerer J, Ewers U, Wilhelm M. Human biomonitoring: state of the art. Int J Hyg Environ Health 2007;210:201-28.

Avlasevich SL, Bryce SM, Cairns SE, Dertinger SD. In vitro micronucleus scoring by flow cytometry: differential staining of micronuclei versus apoptotic and necrotic chromatin enhances assay reliability. Environ Mol Mutagen 2006:47:56-66.

Bastos HN, Antao MR, Silva SN, Azevedo AP, Manita I, Teixeira V, et al. Association of polymorphisms in genes of the homologous recombination DNA repair pathway and thyroid cancer risk. Thyroid 2009;19:1067-75.

Beernink PT, Yang YR, Graf R, King DS, Shah SS, Schachman HK. Random circular permutation leading to chain disruption within and near alpha helices in the catalytic chains of aspartate transcarbamoylase: effects on assembly, stability, and function. Protein Sci 2001;10:528-37.

Berlin A, Schaller KH. European standardized method for the determination of deltaaminolevulinic acid dehydratase activity in blood. Z Klin Chem Klin Biochem 1974;12:389-90.

Bonassi S, Fenech M, Lando C, Lin Y, Ceppi M, Chang WP, et al. HUman MicroNucleus project: international database comparison for results with the cytokinesis-block micronucleus assay in human lymphocytes: I. Effect of laboratory protocol, scoring criteria, and host factors on the frequency of micronuclei. Environ Mol Mutagen 2001;37:31-45

Bravard A, Vacher M, Moritz E, Vaslin L, Hall J, Epe B, et al. Oxidation status of human OGG1-S326C polymorphic variant determines cellular DNA repair capacity. Cancer Res 2009;69:3642-9.
Cao Q Qin C, Meng X, Ju X, Ding Q, Wang M, et al. Genetic polymorphisms in APE1 are associated with renal cell carcinoma risk in a Chinese population. Mol Carcinog 2011;50:863-70.

Cebulska-Wasilewska A. Response to challenging dose of X-rays as a predictive assay for molecular epidemiology. Mutat Res 2003;544:289-97.

Chen L, Elahi A, Pow-Sang J, Lazarus P, Park J. Association between polymorphism of human oxoguanine glycosylase 1 and risk of prostate cancer. J Urol 2003;170:2471-4.

Chen Z, Lou J, Chen S, Zheng W, Wu W, Jin L, et al. Evaluating the genotoxic effects of workers exposed to lead using micronucleus assay, comet assay and TCR gene mutation test. Toxicology 2006;223:219-26.

Conde J, Silva SN, Azevedo AP, Teixeira V, Pina JE, Rueff J, et al. Association of common variants in mismatch repair genes and breast cancer susceptibility: a multigene study. BMC Cancer 2009;9:344.

Ergurhan-Ilhan I, Cadir B, Koyuncu-Arslan M, Arslan C, Gultepe FM, Ozkan G. Level of oxidative stress and damage in erythrocytes in apprentices indirectly exposed to lead. Pediatr Int 2008;50:45-50.

Farinati F, Cardin R, Bortolami M, Nitti D, Basso D, de Bernard M, et al. Oxidative DNA damage in gastric cancer: CagA status and OGG1 gene polymorphism. Int J Cancer 2008;123:51-5.

Figueroa J, Malats N, Real F, Silverman D, Kogevinas M, Chanock S, et al. Genetic variation in the base excision repair pathway and bladder cancer risk. Hum Genet 2007:121:233-42.

García-Lestón J, Méndez J, Pásaro E, Laffon B. Genotoxic effects of lead: an updated review. Environ Int 2010;36:623-36.

García-Lestón J, Roma-Torres J, Vilares M, Pinto R, Cunha LM, Prista J, et al. Biomonitoring of a population of Portuguese workers exposed to lead. Mutat Res 2011;721:81-8.

García-Lestón, J., Roma-Torres, J., Mayan, O., Schroecksnadel, S., Fuchs, D., Moreira, A., et al. Assessment of immunotoxicity parameters in individuals occupationally exposed to lead. J Toxicol Environ Health in press.

García-Quispes WA, Pérez-Machado G, Akdi A, Pastor S, Galofré P, Biarnés F, et al. Association studies of OGG1, XRCC1, XRCC2 and XRCC3 polymorphisms with differentiated thyroid cancer. Mutat Res 2011;709-710:67-72.

Gomes BC, Silva SN, Azevedo AP, Manita I, Gil OM, Ferreira TC, et al. The role of common variants of non-homologous end-joining repair genes XRCC4, LIG4 and Ku80 in thyroid cancer risk. Oncol Rep 2010;24:1079-85.

Grover P, Rekhadevi PV, Danadevi K, Vuyyuri SB, Mahboob M, Rahman MF. Genotoxicity evaluation in workers occupationally exposed to lead. Int J Hyg Environ Health 2010;213:99-106.

Gu D, Wang M, Wang M, Zhang Z, Chen J. The DNA repair gene APE1 T1349G polymorphism and cancer risk: a meta-analysis of 27 case-control studies. Mutagenesis 2009;24:507-12.

Gurer-Orhan H, Sabir HU, Ozgunes H. Correlation between clinical indicators of lead poisoning and oxidative stress parameters in controls and lead-exposed workers. Toxicology 2004;195:147-54.

Hadi MZ, Coleman MA, Fidelis K, Mohrenweiser HW, Wilson III DM. Functional characterization of Ape1 variants identified in the human population. Nucleic Acids Res 2000;28:3871-9.

Hendy GN, Hruska KA, Mathew S, Goltzman D. New insights into mineral and skeletal regulation by active forms of vitamin D. Kidney Int 2006;69(2):218-23.

Hill JW, Evans MK. Dimerization and opposite base-dependent catalytic impairment of polymorphic S326C OGG1 glycosylase. Nucleic Acids Res 2006;34:1620-32.

IARC (International Agency for Research on Cancer). Lead and lead compounds, Inorganic. IARC Monographs, Vol. 23 (Suppl. 7). 1987. Lyon.

IARC (International Agency for Research on Cancer). Inorganic and organic lead compounds. IARC monographs on the evaluation of carcinogenic risks to humans, 87; 2006. Lyon.

Kasuba V, Rozgaj R, Milic M, Zeljezic D, Kopjar N, Pizent A, et al. Evaluation of lead exposure in battery-manufacturing workers with focus on different biomarkers. J Appl Toxicol 2010;30:321-8.

Khan DA, Shazia Q Shahid S, Ansari WM, Khan FA. Lead exposure and its adverse health effects among occupational worker's children. Toxicol Ind Health 2010;26: 497-504.

Kohno T, Kunitoh H, Toyama K, Yamamoto S, Kuchiba A, Saito D, et al. Association of the OGG1-Ser326Cys polymorphism with lung adenocarcinoma risk. Cancer Sci 2006;97:724-8.

Kumar A, Pant MC, Singh HS, Khandelwal S. Role of OGG1 Ser326Cys polymorphism and 8-oxoguanine DNA damage in risk assessment of squamous cell carcinoma of head and neck in north Indian population. Mutat Res 2011;726(2):227-33.

Laantri N, Jalbout M, Khyatti M, Ayoub WB, Dahmoul S, Ayad M, et al. XRCC1 and hOGG1 genes and risk of nasopharyngeal carcinoma in North African countries. Mol Carcinog 2011;50:732-7.

Laffon B, Pásaro E, Méndez J. DNA damage and repair in human leukocytes exposed to styrene-7,8-oxide measured by the comet assay. Toxicol Lett 2002;126:61-8.

Lowry DF, Hoyt DW, Khazi FA, Bagu J, Lindsey AG, Wilson III DM. Investigation of the role of the histidine-aspartate pair in the human exonuclease III-like abasic endonuclease, Ape1. J Mol Biol 2003;329:311-22.

Mansouri MT, Cauli O. Motor alterations induced by chronic lead exposure. Environ Toxicol Pharmacol 2009;27:307-13.

McNeill DR, Narayana A, Wong H-K, Wilson III DM. Inhibition of Ape1 nuclease activity by lead, iron, and cadmium. Environ Health Perspect 2004;112:799-804.

Mendez-Gomez J, Garcia-Vargas GG, Lopez-Carrillo L, Calderon-Aranda ES, Gomez A, Vera E, et al. Genotoxic effects of environmental exposure to arsenic and lead on children in region Lagunera, Mexico. Ann N Y Acad Sci 2008;1140:358-67.

Palus J, Rydzynski K, Dziubaltowska E, Wyszynska K, Natarajan AT, Nilsson R. Genotoxic effects of occupational exposure to lead and cadmium. Mutat Res 2003;540:19-28. 
Pingarilho M, Oliveira NG, Martins C, Gomes BC, Fernandes AS, Martins V, et al.. Sister Chromatid Exchanges induced by acrylamide and glycidamide in human blood cells and relation to polymorphisms in detoxification and repair genes. Toxicology (submitted for publication).

Qian B, Zhang H, Zhang L, Zhou X, Yu H, Chen K. Association of genetic polymorphisms in DNA repair pathway genes with non-small cell lung cancer risk. Lung Cancer 2010;73:138-46.

Roberts MR, Shields PG, Ambrosone CB, Nie J, Marian C, Krishnan SS, et al. Single-nucleotide polymorphisms in DNA repair genes and association with breast cancer risk in the web study. Carcinogenesis 2011;32:1223-30.

Robertson $\mathrm{AB}$, Klungland A, Rognes T, Leiros I. DNA repair in mammalian cells: base excision repair: the long and short of it. Cell Mol Life Sci 2009;66:981-93.

Roddam PL, Rollinson S, O'Driscoll M, Jeggo PA, Jack A, Morgan GJ. Genetic variants of NHEJ DNA ligase IV can affect the risk of developing multiple myeloma, a tumour characterised by aberrant class switch recombination. J Med Genet 2002;39:900-5.

Sakai T. Biomarkers of lead exposure. Ind Health 2000;38:127-42.

Schwartz BS, Hu H. Adult lead exposure: time for change. Environ Health Perspect 2007;115:451-4.

Schwartz BS, McGrail MP, Stewart W, Pluth T. Comparison of measures of lead exposure, dose, and chelatable lead burden after provocative chelation in organolead workers. Occup Environ Med 1994;51:669-73.

Schwartz BS, Stewart WF, Kelsey KT, Simon D, Park S, Links JM, et al. Associations of tibial lead levels with BsmI polymorphisms in the vitamin D receptor in former organolead manufacturing workers. Environ Health Perspect 2000;108:199-203.

Silva SN, Moita R, Azevedo AP, Gouveia R, Manita I, Pina JE, et al. Menopausal age and XRCC1 gene polymorphisms: role in breast cancer risk. Cancer Detect Prev 2007;31: 303-9.

Silva SN, Tomar M, Paulo C, Gomes BC, Azevedo AP, Teixeira V, et al. Breast cancer risk and common single nucleotide polymorphisms in homologous recombination DNA repair pathway genes XRCC2, XRCC3, NBS1 and RAD51. Cancer Epidemiol 2010;34: 85-92.

Smith CC, O'Donovan MR, Martin EA. hOGG1 recognizes oxidative damage using the come assay with greater specificity than FPG or ENDOIII. Mutagenesis 2006;21:185-90.

Tseng RC, Hsieh FJ, Shih CM, Hsu HS, Chen CY, Wang YC. Lung cancer susceptibility and prognosis associated with polymorphisms in the nonhomologous end-joining pathway genes: a multiple genotype-phenotype study. Cancer 2009;115:2939-48.

Uitterlinden AG, Fang Y, Van Meurs JB, Pols HA, Van Leeuwen JP. Genetics and biology of vitamin D receptor polymorphisms. Gene 2004;338:143-56.

Valdiglesias V, Laffon B, Pásaro E, Méndez J. Evaluation of okadaic acid-induced genotoxicity in human cells using the micronucleus test and H2AX analysis. J Toxicol Environ Health A 2011;74:980-92.

Werbrouck J, De Ruyck K, Duprez F, Van Eijkeren M, Rietzschel E, Bekaert S, et al. Singlenucleotide polymorphisms in DNA double-strand break repair genes: association with head and neck cancer and interaction with tobacco use and alcohol consumption. Mutat Res 2008;656:74-81.

Wilson III DM, Kim D, Berquist BR, Sigurdson AJ. Variation in base excision repair capacity. Mutat Res 2011;711:100-12.

Wu PY, Frit P, Meesala S, Dauvillier S, Modesti M, Andres SN, et al. Structural and functional interaction between the human DNA repair proteins DNA ligase IV and XRCC4. Mol Cell Biol 2009;29:3163-72.

Yamane A, Kohno T, Ito K, Sunaga N, Aoki K, Yoshimura K, et al. Differential ability of polymorphic OGG1 proteins to suppress mutagenesis induced by 8-hydroxyguanine in human cell in vivo. Carcinogenesis 2004;25:1689-94.

Ye X, Fu H, Zhu J, Ni W, Lu Y, Kuang X, et al. A study on oxidative stress in lead-exposed workers. J Toxicol Environ Health 1999;56:161-72. 